\title{
Proteome Profile of Muscle Tissue of Indian Walking Catfish, Clarias Magur Exposed to Abiotic Temperature Stress
}

Poonam Jayant Singh ( $\sim$ poonamjayant@gmail.com )

NBFGR: National Bureau of Fish Genetic Resources https://orcid.org/0000-0002-9476-644X

Satish Kumar Srivastava

NBFGR: National Bureau of Fish Genetic Resources

\section{Research Article}

Keywords: Proteome, Liquid Chromatography -Mass Spectroscopy, abiotic stress, Clarias magur

Posted Date: December 15th, 2021

DOI: https://doi.org/10.21203/rs.3.rs-1134287/v1

License: (c) (1) This work is licensed under a Creative Commons Attribution 4.0 International License.

Read Full License 


\section{Abstract}

The study of expression of proteins in organisms on exposure to various environmental challenges gives clues for understanding on how these challenges affects and copes with the biological system. A study was undertaken to understand the proteome profile of Clarias magur, exposed to abiotic stress of water temperature, to find how fishes evolve adaptive strategies towards stress induced by unforeseen vagaries of climate change. Specimens of Clarias magur were exposed to high temperature sub-lethal water stress of $37^{\circ} \mathrm{C}$ for 60 days and the muscle proteome profiling was analysed through Liquid Chromatography -Mass Spectroscopy for qualitative differential profiling. The study provides an understanding of different proteins expressed as adaptative challenge to the environment. This is the first study to see proteome expression in Clarias magurthrough Liquid Chromatography -Mass Spectroscopy

\section{Introduction}

Climate change is a major contributory factor towards adaptation of ecosystems from where the food emerges. UN Sustainable Development Goals (SDGs), particularly the SDG1 (end poverty), SDG2 (end hunger), SDG13 (good health and well-being) and SDG4 (action for reducing impact of climate change) has direct linkage to provide an integrative framework for bringing together the policy and the system initiatives to counter the vagaries of climate change. Climate change shall eventually impact food production due to rising temperature, affecting the adaptability of the genetic resources and their productivity. By the middle of $21^{\text {st }}$ century, the global population of $\sim 9$ billion people will be ready to be fed. The Paris Agreement of the United Nations Framework Convention on Climate Change (UNFCCC), which aims to keep the global temperature rise this century well below $2^{\circ} \mathrm{C}$ above pre-industrial levels, recognizes the fundamental priority of safeguarding the food security to integrate the climate change measures into national policies, strategies and planning to reduce the effects of climate change on the productivity. Aquaculture heavily depends on ambient environment, hence vulnerable to climate change, potential of epigenetic adaptation can improve stress tolerance through early life exposure for which real time complex data is needed on multiple stressor levels for prediction of long term change for planning in response to climate change adaptation and mitigation [ [ $]$. Aquaculture has adapted its self at local level, by changing cultural practices and community based adaptive planning as identified through a study published from 1990 to 2018 [2]. United Nations 2030 Agenda supports transformation to a sustainable future while FAO fights with hunger and poverty, innovation and new design solutions are needed to get solutions. Aquaculture survives in varied locations with different environmental and diverse climatic factors. Though FAO does not keep pace with actual species diversification in aquaculture, it shows an increase from 472 species in 2006 to 622 species in 2018. Around 27 finfish species accounted for 90 percent of total finfish production in 2018[3]. Nadarajah and Eide, $2020\left[{ }^{4}\right]$ evaluated relisence of shrimp, tilapia, carp and catfish in sequence, indicating high diversity with high adaptation capacity specially in Asia that has capacity to adapt with proper modifications in farming system in future. Climate change also affects temperate region, where mitigation strategy would be to adapt existing species, wild and domesticated for temperate temperature tolerance along with collaboration with stakeholders, 
researchers and government shall help in developing mitigating strategies for climate change $\left.{ }^{5}\right]$. A metaanalysis study found that aquatic animals present higher mortalities at warmer temperatures induced due to climate change, also making them susceptible to high antimicrobial resistance $\left.{ }^{6}\right]$. Providing an environment that is stress free for aquaculture shall have its benefits in long term, considering minimum stress and maximum welfare for the aquaculture. Fishes when stressed jeopardises their health and survival, while adjusting to the aquatic conditions $\left[{ }^{7}\right]$. Chemical imbalances in water cause direct harm to fish by disrupting physiological functions as ionic regulation, gill and kidney function, by destroying the fishes' mucous coating $\left[{ }^{8}\right]$,which is a primary protection against pathogenic and parasitic invasion $\left[{ }^{9}\right]$. The facultative air breathing catfish, Clariasmagur (magur), has more commercial importance as food fish that can thrive in swampy water conditions where many teleosts cannot survive due to intolerance to high environmental ammonia. An initiative was taken to analyse stress induced changes in the fish proteome by exposing them to the sub lethal high temperature. The peptides from the Liquid Chromatography Mass Spectrometry (LC-MS) were analysed to study the effect on the adaptability of C.magur to this temperature stress as preparedness for tolerance induced by the climate change. The primary aim of the study was to understand adaptive mechanism undergoing at genomic and transcriptomic level ultimately translating to proteomic level. The complexity of the system was frozen after a time span established under experimental conditions to understand basic proteome changes underlying a stressful condition. The study was taken up to understand the complexity of the physiological changes starting with activation of sympathetic response altering the energy metabolism. In the downstream process, energy is metabolised that is coped by the activation of the glycogenolysis and gluconeogenesis from muscle or liver $\left.{ }^{[10}\right]$. Lactate can be generated from the muscle due to external stressful stimuli leading to anaerobic glycolysis, which is released into plasma $\left[{ }^{11}\right]$. The stress induction leads to alterations in the overall performance impacting growth, immune response and health $\left[{ }^{12}\right]$. Proteomics has emerged as a powerful tool towards the deep understanding of the fish biology, helping aquaculture to reach its main goal of high productivity with better quality products. The conduct of experiment is represented in graphical abstract Figure 1. Compared to genomics, the proteome provides not only information at a mechanistic level but can also captures the changes in protein activity measured as post-translational modifications. It deciphers the amount of protein present in an organism at a particular time, thus, helps to analyse the responses from the expressed proteome profile. Whereas genome remains the blue print, protein expressed helps in adapting to changing environmental and anthropometric stress.

\section{Materials And Methods}

Healthy live specimens $(n=15)$ of Clarias magur (weighting 50-90 g) were procured from the local area in and around Lucknow, Uttar Pradesh, India. The specimens were anaesthetised in clove oil and transported to indoor hatchery in 150 litre plastic tank with proper oxygenation. The fishes were then acclimatized in a re-circulatory system for 15 days at $26^{\circ} \mathrm{C}$. After acclimatization, the specimens were randomly divided into control and experimental groups. Each group contained 10 fishes. The control group were kept at ambient water temperature of $26^{\circ} \mathrm{C}$ in re-circulatory system, while experimental were 
exposed to $37^{\circ} \mathrm{C}$ sub lethal limit by increasing the water temperature $1^{\circ} \mathrm{C}$ per day from the initial temperature. Both the groups were exposed for long duration of 60 days, and were monitored daily. After experimentation, 3 specimens from both groups were sacrificed under anaesthetic condition with clove oil. Muscle tissues samples were dissected and immediately frozen in liquid nitrogen and also kept at $-80^{\circ} \mathrm{C}$ till further protein extraction. The fishes were taken care of ethically by anesthetising with clove oil before dissection

\section{Protein extraction and LC MS}

Protein was extracted from the stored muscle samples of 3 control and 3 experimental groups within a week of conducting the experiment. Briefly, the samples were grinded and homogenised in $50 \mathrm{mM}$ Tris Buffer containing protease inhibitor cocktail and phenylmethylsulfonyl fluoride (PMSF), centrifuged at 14,000 rpm to remove the debris. The supernatant was collected in Tris buffer extract and run on SDSPAGE to check quality for downstream processing. The protein was quantified by BCA method. $100 \mu \mathrm{g}$ of extracted protein was processed for running in LC-MS. The sample was treated with $100 \mathrm{mM}$ dithiothreitol (DTT) for $1 \mathrm{~h}$ at $95^{\circ} \mathrm{C}$ for reducing disulphide bonds of cysteine, followed by treatment with $250 \mathrm{mM}$ IDA for $45 \mathrm{~min}$ at room temperature in dark to alkylate cystine formed after reduction of the cysteine. The sample was then digested with trypsin, which specifically cleaves at lysine and arginine amino acids, and incubated overnight at $37^{\circ} \mathrm{C}$. The peptides from the sample were extracted in $0.1 \%$ formic acid and incubated for $45 \mathrm{~min}$ at $37^{\circ} \mathrm{C}$ followed by centrifugation at $10,000 \mathrm{~g}$. The supernatant was collected and dried in vacuum. The dried sample was dissolved in $20 \mu \mathrm{l}$ of $0.1 \%$ formic acid in water. This sample was then used for injecting in LC-MS. $10 \mu \mathrm{L}$ injection volume was used on Ethylene Bridged Hybrid $\mathrm{C}_{18}$ nano columns (Hydrophobic stationary phase) of UPLC for separation of peptides, based on their affinity, with hydrophilic molecules eluting first. The peptides separated on the column were directed to Waters Synapt G2 Q-TOF instrument for Data Independent Acquisition (DIA) for MS and MS/MS analyses. The raw data was processed by Mass Lynx 4.1 WATERS. The individual peptides MS/MS spectra were matched to the database sequence for protein identification on PLGS software (Protein Lynx Global Server), WATERS with UniProt (https://www.uniprot.org/)reviewed sequences of zebrafish. The raw data of elutant and mass/ charge value was subjected to PLGS software and data was processed with UniProt (https://www.uniprot.org/) reviewed proteins of zebrafish. Qualitative differential expression of proteins was based on $50 \mathrm{ppm}$ peptide tolerance, $100 \mathrm{ppm}$ fragment tolerance with minimum fragment match of 2 peptides for proteins. Peptides were modified (carbamidomethylation and oxidation) and the retrieved proteins were analysed, visualised and interpreted. 25 most relevant pathways, sorted by p-values, were analysed in control and experimental groups.

C. magurfishes were exposed to abiotic temperature stress and Liquid chromatography was performed on a ACQUITY UPLC system (Waters). The separation of all samples was performed on ACQUITY UPLC BEH C18 column (Waters) $(150 \mathrm{~mm} \times 2.1 \mathrm{~mm} \times 1.7 \mu \mathrm{m})$. A gradient elution program was run for the chromatographic separation with mobile phase A (0.1\% Formic Acid in WATER), and mobile phase B ( $0.1 \%$ formic Acid in acetonitrile) was programmed. A SYNAPT G2 QTOF (Waters) equipped with an Electro-Spray lonization (ESI) source was used for mass spectrometric detection. ESI is a soft ionisation 
technique that sprays sample through ESI capillary at atmospheric pressure. Sample analysis was performed in positive mode. The Time of Flight (TOF) was set for the range of $50 \mathrm{Dalton}(\mathrm{Da})$ to $1500 \mathrm{Da}$ with a scan time of 0.5 second. The raw data acquired from the machine was processed using PLGS software 3.0.2 where Data Processing and Database Search was performed. The proteome dataset was not available, as the source sample being $C$. magur, hence, a more annotated species zebrafish was used to compare FASTA sequences. The protein sequences in FASTA format were downloaded from the SwissProt database and used for searching the peptides present in the samples. One run of each sample was processed with $50 \mathrm{ppm}$ Peptide Tolerance, 100 ppm Fragment Tolerance, 2 as Minimum number of Fragment matches for Peptides, minimum number of Fragment matches for Proteins being one missed cleavage and fixed modification of cysteine carbamidomethylation and variable peptide modification were oxidation of methionine and deamination of glutamine and asparagine in the search engine PLGS. The data was analysed from experimental and control samples for gene ontology and pathway analysis.

\section{Data Availibility}

The mass spectrometry proteomics raw experimental data has been deposited to the ProteomeXchange Consortium via the PRIDE ${ }^{13}$ partner repository with the dataset identifier PXD029636 and DOI 10.6019/PXD029636. The control data is available via ProteomeXchange with identifier PXD028115. Experimental data is available via ProteomeXchange with identifier PXD029636 with project name as Identification of Protein Profile in Muscle Tissue of Threatened Indian walking catfish, Clarias magur (Hamilton 1822).

\section{Data Analysis}

\section{Identification of proteins:}

The peptide spectra was identified by its PLGS Score, that uses a Monte Carlo algorithm to analyse all available mass spectra data, providing a statistical measure of accuracy of assignation with a higher score implying a greater confidence of protein identity $\left[{ }^{14},{ }^{15}\right]$. The identified proteins from experimental and control groups were analysed for their significance and regulatory mechanism. A representative chromatogram and peptide mass/charge ratios are shown in Figure 2 showing chromatogram of elutant and mass spectra of control and experimental samples.

The differential expression of proteins retrieved from the muscle of $C$. magur exposed to high water temperature stress was analysed. Since the peptides analysed were not same in all three samples due to variation in the protein profile at a time point, which could be attributable to various factors like growth, adaptability differences etc. Retrieved peptides were analysed, visualised and interpreted with pathway database against the zebrafish. A total of 1570 common proteins were expressed in both the control and experimental groups. On the other hand, 310 differentially expressed proteins were found in experimental and 589 proteins were found in control groups. In control samples 1585, 1199, 1201 proteins were expressed respectively of which 581 were expressed in all samples. Single unique proteins expressed 
were 2159 in number. In experimental samples 1225, 1061, 1061 proteins were expressed respectively of which 437 were expressed in all samples. Single unique proteins expressed were 1880 in number. On curation of protein elements only those proteins were taken into consideration that were expressed in all control samples and all experimental samples to find uniform minimum expression of common proteins. Similar proteins were taken into consideration for experimental samples. 581 proteins were expressed in all control samples, and 437 proteins were expressed in all experimental samples. The common proteins in experimental and control samples compared together was 275 , differential expressed proteins were 163, while 306 proteins (Figure 3 ) were expressed in control samples only.

\section{Results And Discussion}

The objective of the study was to understand changes occurring in the metabolism of fish when exposed to temperature stress. The study throws light on the differential expression of proteins under stress. During normal conditions the common proteins show fishes undergoing gluconeogenesis that only happens during the starvation conditions, when energy in the body is low and the fish uses stored energy. Differential expression of proteins show how fish adapts to environmental changes in fish through metabolism at cellular level. The 25 most relevant pathways sorted by $p$-value for proteins expressed in experimental fishes from 163 proteins expressed predominantly belonging to the Platelet endothelial cell adhesion molecule-1 (PECAM-1) interactions. (PECAM-1 is a member of the immunoglobulin (Ig) and is a key participant in the adhesion cascade leading to extravasation of leukocytes during the inflammatory process ${ }^{16}$ (Newman, P.J., 1997). The pathways differentially expressed were DCC mediated attractive signalling pathway, (DCC mediated attractive signalling pathway includes DCC and neogenin in vertebrates), regulation of KIT signalling (KIT is a cytokine receptor expressed on the surface of hematopoietic stem cells as well as other cell types. Altered forms of this receptor may be associated with some types of cancer [ $\left.{ }^{17}\right]$, Abasic sugar-phosphate removal via the single-nucleotide replacement pathway (Apurinic/apyrimidinic endonuclease 1 (APE1) acts as a reductive activator of many transcription factors (TFs) and is involved in the base excision repair (BER) pathway, which repairs oxidative base damage caused by endogenous and exogenous agents. APE1 activates activator protein1 , nuclear factor kappa B, hypoxia-inducible factor $1 \mathrm{a}$, paired box gene 8 , signal transducer activator of transcription 3 and p53, which are involved in apoptosis, inflammation, angiogenesis and survival pathways. APE1/Ref-1 maintains cellular homeostasis (redox) via the activation of TFs that regulate various physiological processes and that crosstalk with redox balancing agents by controlling levels of reactive oxygen and nitrogen species $\left[{ }^{18}\right]$. Abasic sugar phosphate removal via the single nucleotide replacement pathway requires displacement of DNA glycosylase by apurinic/apyrimidinic endodeoxyribonuclease 1 (APEX1), APEX1-mediated endonucleolytic cleavage at the 5 ' side of the base free deoxyribose residue, recruitment of DNA polymerase beta (POLB) to the apurinic/apyrimidinic (AP) site and excision of the abasic sugar phosphate ( $\left.5^{\prime} \mathrm{dRP}\right)$ residue at the strand break $\left[{ }^{19}\right]$. The two major activities of APEX1 multifunctional protein are DNA repair and redox regulation of transcriptional factors. It functions as a apurinic/apyrimidinic (AP) endodeoxyribonuclease in the DNA base excision repair (BER) pathway of DNA lesions induced by oxidative and alkylating agents and possesses a DNA 3' 
phosphodiesterase activity capable of removing lesions. May also play a role in the epigenetic regulation of gene expression by participating in DNA demethylation and plays a role in the protection from granzymes-mediated cellular repair leading to cell death. Also involved in the DNA cleavage step of class switch recombination (CSR). The following protein pathways were involved in stress regulation viz, EPHA-mediated growth cone collapse, ephrin signalling, regulation of gap junction activity, activated NTRK3 signals through PI3K, adrenaline signalling through Alpha-2 adrenergic receptor, metalloprotease DUBs, resolution of AP sites via the single nucleotide replacement pathway, signalling by NTRK3 (TRKC), fructose catabolism, NTRK2 activates RAC1, activated NTRK2 signals through FYN, eukaryotic translation elongation, reelin signalling pathway, molybdenum cofactor biosynthesis, Vitamin D (calciferol) metabolism, nuclear receptor transcription pathway, deubiquitination, Netrin-1 signaling, APEX1-Independent resolution of AP sites via the single nucleotide replacement pathway, regulation of granulopoiesis and synthesis of dolichyl-phosphate.

The 25 most relevant pathways for proteins expressed differentially in control fishes from 306 proteins expressed predominantly belong to be associated with the pathways associated with synthesis of PIPS at the ER membrane, regulation of TP53 expression, VEGF ligand-receptor interactions, , receptor dimerization, release of $\mathrm{Hh}-\mathrm{Np}$ from the secreting cell, transcriptional activation of p53 responsive genes, transcriptional activation of cell cycle inhibitor p21, regulation of TP53 activity through methylation, synthesis of PE, oncogene induced senescence, synthesis of PIPs at the golgi membrane, FGFR1c and klotho ligand binding and activation, Beta Klotho-mediated ligand binding, Proton/ oligopeptide cotransporters, $\mathrm{N}$-glycan trimming in ER and Calnexin/ Calreticulin cycle, transport of mature mRNA derived from an Intron-Containing Transcript, transport of mature transcript to cytoplasm, mRNA 3'-end processing, RNA Polymerase II transcription termination, Invadopodia formation, Zinc efflux and compartmentalization by the SLC30 family, synthesis of IPs in nucleus, MASTL facilitates mitotic progression, trafficking of myristoylated proteins to cilium, FGFR1b ligand binding and activation.

The 25 most relevant pathways sorted by p-value for proteins commonly expressed in control and experimental fishes from 275 proteins expressed predominantly belong to be associated with SEMA3APlexin repulsion signalling by inhibiting integrin adhesion, Sema3A PAK dependent Axon repulsion, CRMPs in Sema3A signalling, VEGFR2 mediated cell proliferation, crosslinking of collagen fibrils, PECAM1 interactions, metalloprotease DUBs, abasic sugar-phosphate removal via single-nucleotide replacement pathway, gluconeogenesis, unblocking of NMDA receptors, glutamate binding and activation, activation of Ca-permeable Kainate receptor, DCC mediated attractive signalling, ionotropic activity of kainate receptors, regulation of KIT signalling, RHO GTPases regulate CFTR trafficking, regulation of gap junction activity, activated NTRK3 signals through PI3K, adrenaline signalling through Alpha-2 adrenergic receptor, anchoring fibril formation, MET activates PTK2 signalling, resolution of AP sites via single-nucleotide replacement pathway, TFAP2 (AP-2) family regulates transcription of cell cycle factors, signalling by NTRK3 (TRKC), antagonism of activin by Follistatin neurophilin interactions with VEGF and VEGFR. 
Semaphorins are extracellular signaling proteins characterized by a single cysteine-rich extracellular sema domain, function as axon guidance molecules, but it is now understood that semaphorins, are key regulators of morphology and Semaphorin signaling occurs predominantly through Plexin receptors and results in changes to the cytoskeletal and adhesive machinery that regulate cellular morphology $\left[{ }^{20}\right]$. Sema3A, a prototypical semaphorin, acts as a chemorepellent or a chemoattractant for axons by activating a receptor complex comprising neuropilin-1 as the ligand-binding subunit and plexin-A1 as the signal-transducing subunit. FARP2 is a key molecule involved in the response of neuronal growth cones to class-3 semaphorins $\left[{ }^{21}\right]$. The members of the collapsin response mediator protein (CRMP) family-five cytosolic phosphoproteins-are highly expressed throughout brain development. Moreover, the expression of CRMPs is altered in neurodegenerative diseases, and these proteins may be of key importance in the physiopathology of the adult nervous system [22]. Vascular endothelial growth factor (VEGF) and VEGF receptor 2 (VEGFR2) are increased in majority species of cancers and suppress tumor progression by blocking VEGF/VEGFR2. Apatinib is a highly selective VEGFR2 antagonist which has inhibitive effect on antiapoptotic and cell growth in CCA. While, the effect of apatinib cell migration and invasion in CCA is still unknown $\left[{ }^{23}\right]$. PECAM-1/CD31 is a member of the immunoglobulin superfamily (IgSF) and has been implicated to mediate the adhesion and trans-endothelial migration of T-lymphocytes into the vascular wall, T cell activation and angiogenesis. For 275 Commonly Expressed Proteins (CEP), in experimental and control samples, gene ontology, enzyme class and pathway was analyzed by comparing proteins with curated zebrafish UniPort database with reviewed Swiss-Prot database. Similar analysis was performed for 163 Differentially Expressed Proteins (DEP) in experimental samples. The Molecular Function, Cellular Component and Biological process of 275 Commonly Expressed Proteins in control and experimental samples of muscle tissue exposed to temperature stress as depicted in Figure 4 shows more proteins involved in biological process. Enzymatic activity of 275 Commonly Expressed Proteins (CEP) in control and experimental samples of muscle tissue exposed to temperature stress is shown in Figure 5, depicting oxidoreductases, transferases, hydrolases and enzymes altering polypeptide conformation. Figure 6 shows Pathway analysis of 275 Commonly Expressed Proteins in control and experimental samples of muscle tissue exposed to temperature stress with major proteins involved in protein modification mainly ubiquitination and glycosylation. Figure 7 shows Gene Ontology of 581 proteins expressed only in Control Samples of Muscle tissue. Figure 8 shows Gene ontology of 163 proteins expressed in experimental samples of muscle tissue exposed to temperature stress. Figure 9 depicts enzymatic analysis of 163 proteins expressed in experimental muscle tissue samples exposed to temperature stress. Figure 10 depicts pathway analysis of 163 proteins expressed in experimental muscle tissue samples exposed to temperature stress

The major pathway of proteins expressed in response to high temperature stress was related to protein modifications glycosylation and ubiquitination. Ubiquitination is a multistep exzymatically catalyzed post-translational modification process that targets proteins for degradation and recycling . Ubiquitination is critical in almost every cellular process, has a role in modulating diverse cellular functions like cell proliferation and differentiation, autophagy, apoptosis, immune response, DNA repair, neural degeneration, myogenesis, and stress response as well as a major player in almost any disease or 
disorder $[24,25,26,27,28]$. The pathway analysis and enzymatic activity of differentially expressed proteins provide a glimpse of the strategy used by Clarias magur for adapting for developing resilience for climate change. Further quantitative study can be helpful for understanding micro level interaction of proteins. This study provides a glimpse into the adaptive strategy used by Clarias magur for adaptation for temperature stress.

\section{Declarations}

\section{Authors' declaration:}

\section{Contribution: Paper writing and analysis- Dr Poonam Jayant Singh,}

\section{Experiment Conduct: Dr Satish Kumar Srivastava}

Acknowledgement: The authors express thanks to the Director, ICAR-National Bureau of Fish Genetic Resources, Lucknow for facilitating the work. The work has been carried out as a part of the ICAR-NBFGR institute funded project "Stress tolerance of prioritised fish species" with grant number FISHNBFGR51L201700200191. The authors declare no conflict of Interest.

\section{Data availability}

The raw data has been submitted to PRIDE The mass spectrometry proteomics raw experimental data has been deposited to the ProteomeXchange Consortium via the PRIDE. partner repository with the dataset identifier PXD029636 and DOI 10.6019/PXD029636. The control data is available via ProteomeXchange with identifier PXD028115. Experimental data is available via ProteomeXchange with identifier PXD029636 with project name as Identification of Protein Profile in Muscle Tissue of Threatened Indian walking catfish, Clarias magur (Hamilton 1822). Additional data supporting the findings of this study are available from the corresponding author upon reasonable request.

\section{Funding}

Funding for the project FISHNBFGRSIL2017002191 was recieved from ICAR under institutional grant

\section{Conflicts of interest/Competing interests}

The authors declare that there is no financial or personal interest or belief that could affect their objectivity, or if there is, stating the source and nature of that potential conflict

\section{Ethics approval/declarations :}

Ethical approval was obtained from ICAR-NBFGRs Institutional committee

Consent to participate Not applicable 
Consent for publication The consent to be a part of the publication has been provided by all authors

Code availability (software application or custom code) not applicable

Authors' contributions: PJS wrote the paper, analysed data, investigated formal analysis with interpretation with methodology and writing SKS conducted the experiment, coordination and paper writing. All authors read and approved the final manuscript

\section{References}

1. Reid, G.K., Gurney-Smith, H.J., Flaherty, M., Garber, A.F., Forster, I., Brewer-Dalton, K., Knowler, D., Marcogliese, D.J., Chopin, T., Moccia, R.D. and Smith, C.T., 2019. Climate change and aquaculture: considering adaptation potential. Aquaculture Environment Interactions, 11, pp.603-624.

2. Galappaththi, E. K., Ichien, S. T., Hyman, A. A., Aubrac, C. J., \& Ford, J. D. (2020). Climate change adaptation in aquaculture. Reviews in Aquaculture, 12(4), 2160-2176.

3. FAO. 2020 Food and Agriculture organisation of the United Nations report on "The State of World Fisheries and Aquaculture " 2020. Sustainability in action. Rome. Report available at https://doi.org/10.4060/ca9229en from website http://www.fao.org/state-of-fisheries-aquaculture (accessed 16 June 2021)

4. Nadarajah, Suthamathy, and Arne Eide. "Are Asian fresh and brackish water aquaculture production vulnerable or resilient towards climate change impacts?." Aquaculture Economics \& Management 24, no. 3 (2020): 232-254.

5. Bricknell, I.R., Birkel, S.D., Brawley, S.H., Van Kirk, T., Hamlin, H., Capistrant-Fossa, K., Huguenard, K., Van Walsum, G.P., Liu, Z.L., Zhu, L.H. and Grebe, G., 2020. Resilience of cold water aquaculture: a review of likely scenarios as climate changes in the Gulf of Maine. Reviews in Aquaculture.

6. Reverter, M., Sarter, S., Caruso, D., Avarre, J.C., Combe, M., Pepey, E., Pouyaud, L., Vega-Heredía, S., De Verdal, H. and Gozlan, R.E., 2020. Aquaculture at the crossroads of global warming and antimicrobial resistance. Nature communications, 11(1), pp.1-8.

7. Conte, F. S. Stress and the welfare of cultured fish. Appl. Anim. Behav. Sci. 86, 205-223 (2004)

8. Post, G.B., 1987. Text Book of Fish Diseases. TFH Publications, Neptune, New Jersey, p. 287

9. Klontz, G.W., 1993. Environmental requirements and environmental diseases of salmonids. In: Stoskopf, M. (Ed.), Fish Medicine. Saunders, Philadelphia, PA, USA, pp. 333-342.

10. Vijayan, M. M., Aluru, N. \& Leatherland, J. F. Stress response and the role of cortisol. Fish Dis. Disord.2, 182-201 (2010).

11. Milligan, C. L. \& Girard, S. S. Lactate metabolism in rainbow trout. J. Exp. Biol. 180, 175-193 (1993).

12. Boonstra, R. Reality as the leading cause of stress: rethinking the impact of chronic stress in nature. Funct. Ecol.27, 11-23 (2013).

13. Perez-Riverol Y, Csordas A, Bai J, Bernal-Llinares M, Hewapathirana S, Kundu DJ, Inuganti A, Griss J, Mayer G, Eisenacher M, Pérez E, Uszkoreit J, Pfeuffer J, Sachsenberg T, Yilmaz S, Tiwary S, Cox J, 
Audain E, Walzer M, Jarnuczak AF, Ternent T, Brazma A, Vizcaíno JA (2019). The PRIDE database and related tools and resources in 2019: improving support for quantification data. Nucleic Acids Res 47(D1):D442-D450 (PubMed ID: 30395289).

14. Rosenegger, D., Wright, C. \& Lukowiak, K. A quantitative proteomic analysis of long-term memory. Mol. Brain3, 9 (2010).

15. Wright, C. et al. Ankylosing spondylitis monocytes show upregulation of proteins involved in inflammation and the ubiquitin proteasome pathway. Ann. Rheum. Dis.68, 1626-1632 (2009).

16. Newman, P.J., 1997. The biology of PECAM-1. The Journal of clinical investigation, 99(1), pp.3-8.

17. Edling, C.E. and Hallberg, B., 2007. c-Kit-a hematopoietic cell essential receptor tyrosine kinase. The international journal of biochemistry \& cell biology, 39(11), pp.1995-1998.

18. Thakur, S., Sarkar, B., Cholia, R.P., Gautam, N., Dhiman, M. and Mantha, A.K., 2014. APE1/Ref-1 as an emerging therapeutic target for various human diseases: phytochemical modulation of its functions. Experimental \& molecular medicine, 46(7), pp.e106-e106.

19. Lindahl, T. and Wood, R.D., 1999. Quality control by DNA repair. Science, 286(5446), pp.1897-1905.

20. Alto, L.T. and Terman, J.R., 2017. Semaphorins and their signaling mechanisms. In Semaphorin Signaling (pp. 1-25). Humana Press, New York, NY.

21. Toyofuku, T., Yoshida, J., Sugimoto, T., Zhang, H., Kumanogoh, A., Hori, M. and Kikutani, H., 2005. FARP2 triggers signals for Sema3A-mediated axonal repulsion. Nature neuroscience, 8(12), pp.17121719.

22. Charrier, E., Reibel, S., Rogemond, V., Aguera, M., Thomasset, N. and Honnorat, J., 2003. Collapsin response mediator proteins (CRMPs). Molecular neurobiology, 28(1), pp.51-63.

23. Huang, M., Huang, B., Li, G. and Zeng, S., 2018. Apatinib affect VEGF-mediated cell proliferation, migration, invasion via blocking VEGFR2/RAF/MEK/ERK and PI3K/AKT pathways in cholangiocarcinoma cell. BMC gastroenterology, 18(1), p.169.

24. Rechsteiner, M. (1987). Ubiquitin-mediated pathways for intracellular proteolysis. Annual review of cell biology, 3(1), 1-30.

25. Shi, C. S., \& Kehrl, J. H. (2010). TRAF6 and A20 regulate lysine 63-linked ubiquitination of Beclin-1 to control TLR4-induced autophagy. Science signaling, 3(123), ra42-ra42.

26. Jin, X., Cheng, H., Chen, J., \& Zhu, D. (2011). RNF13: an emerging RING finger ubiquitin ligase important in cell proliferation. The FEBS journal, 278(1), 78-84.

27. Bosanac, I., Wertz, I.E., Pan, B., Yu, C., Kusam, S., Lam, C., Phu, L., Phung, Q., Maurer, B., Arnott, D. and Kirkpatrick, D.S., 2010. Ubiquitin binding to A20 ZnF4 is required for modulation of NF-KB signaling. Molecular cell, 40(4), pp.548-557.

28. Karve, T. M., \& Cheema, A. K. (2011). Small changes huge impact: the role of protein posttranslational modifications in cellular homeostasis and disease. Journal of amino acids, 2011.

\section{Figures}




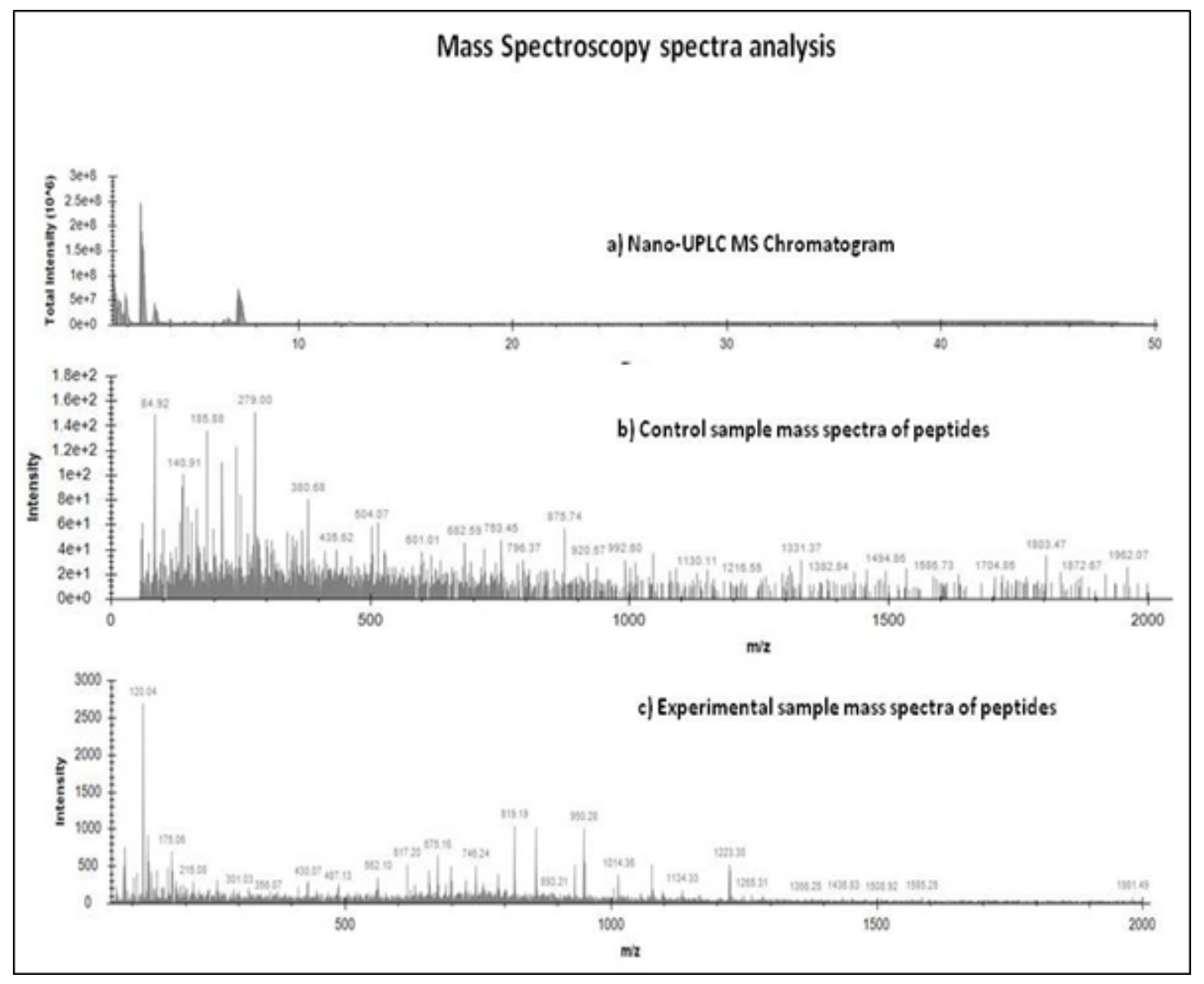

\section{Figure 1}

(a)Nano-UPLC Chromatogram of elutant; (b)Representative Mass spectra of tryptic peptides of control sample; (c) Representative Mass spectra of tryptic peptides of experimental sample 


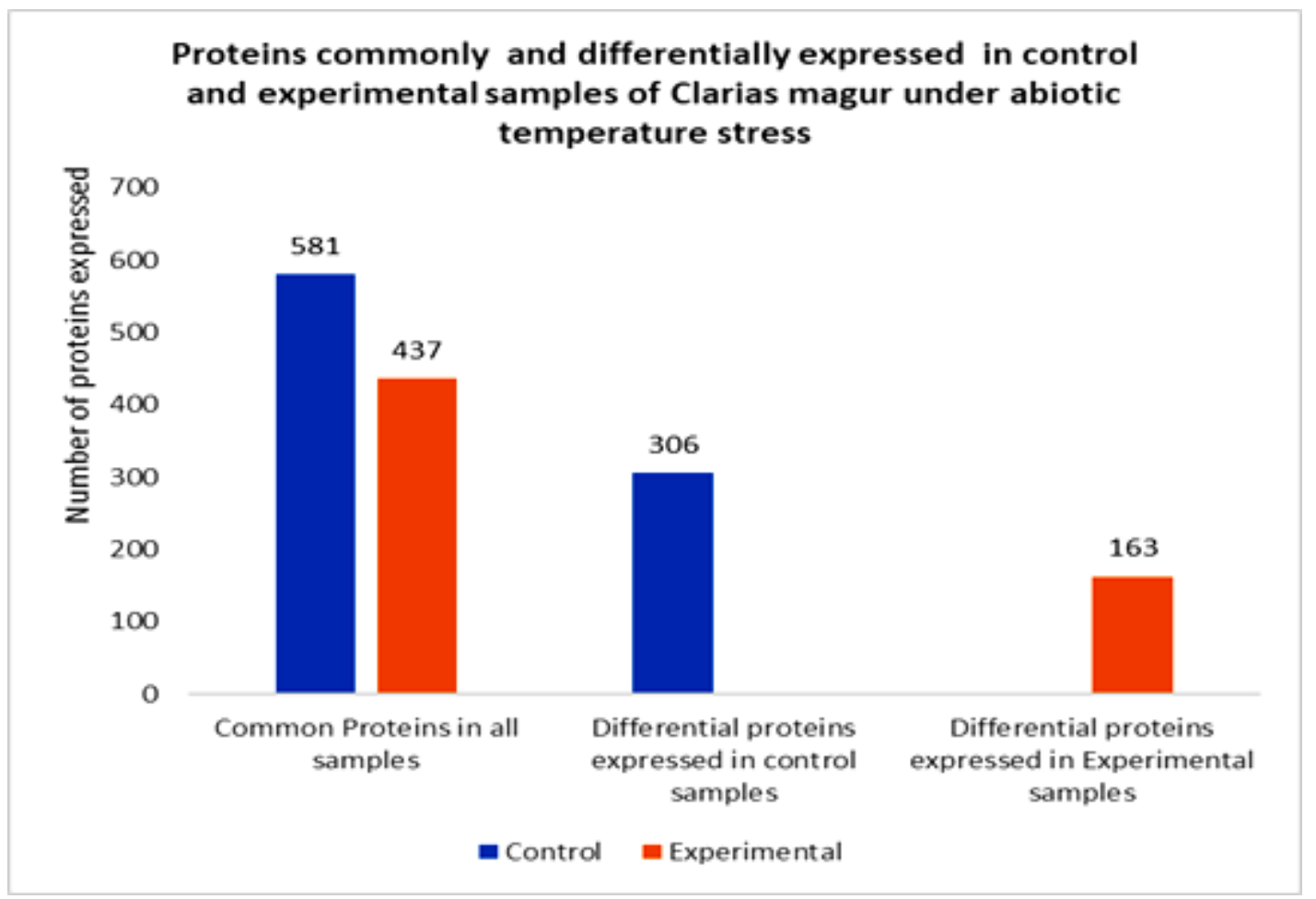

Figure 2

Number of Proteins commonly and differentially expressed in control and experimental samples

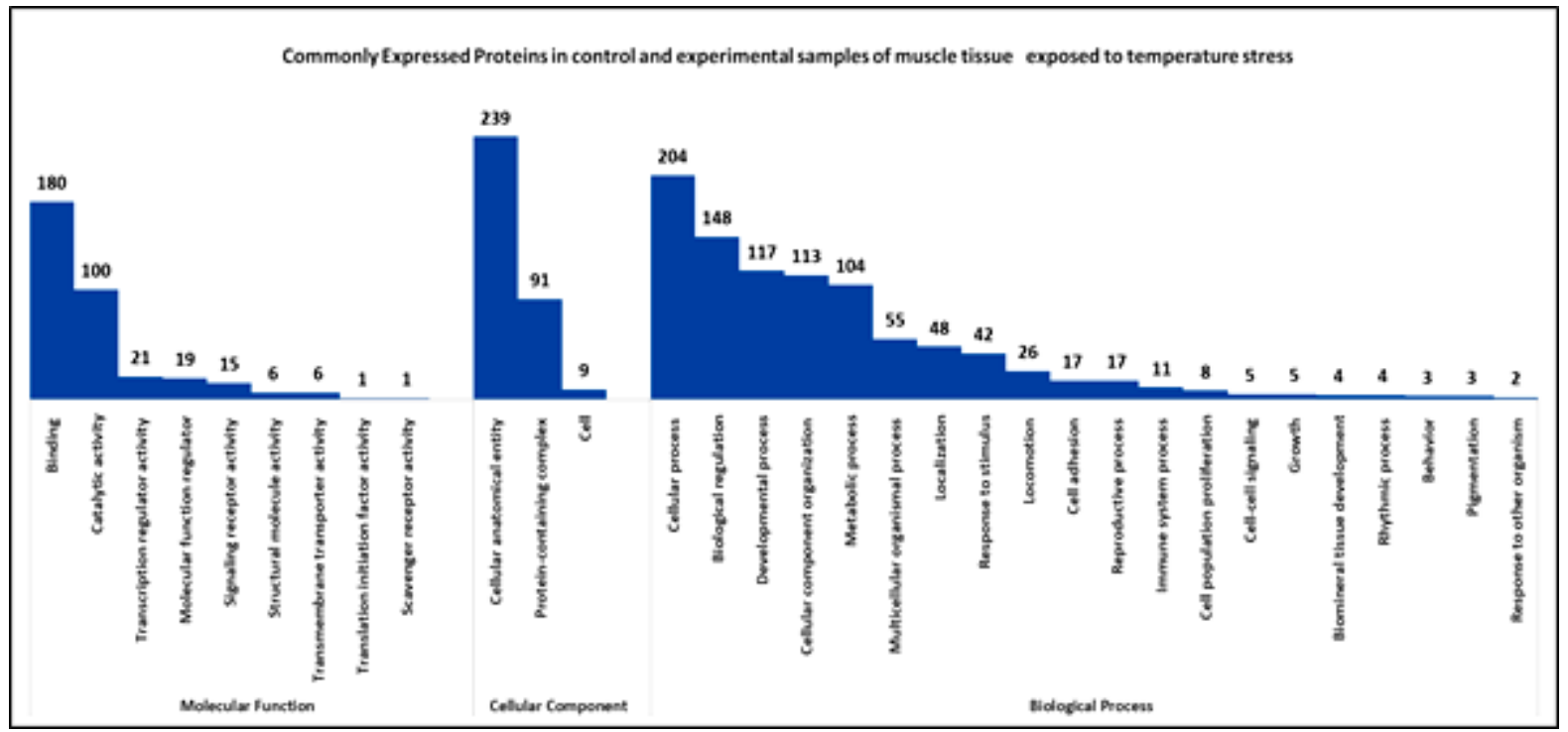

Figure 3

Molecular Function, Cellular Component and Biological process of 275 Commonly Expressed Proteins in control and Experimental samples of muscle tissue exposed to temperature stress 


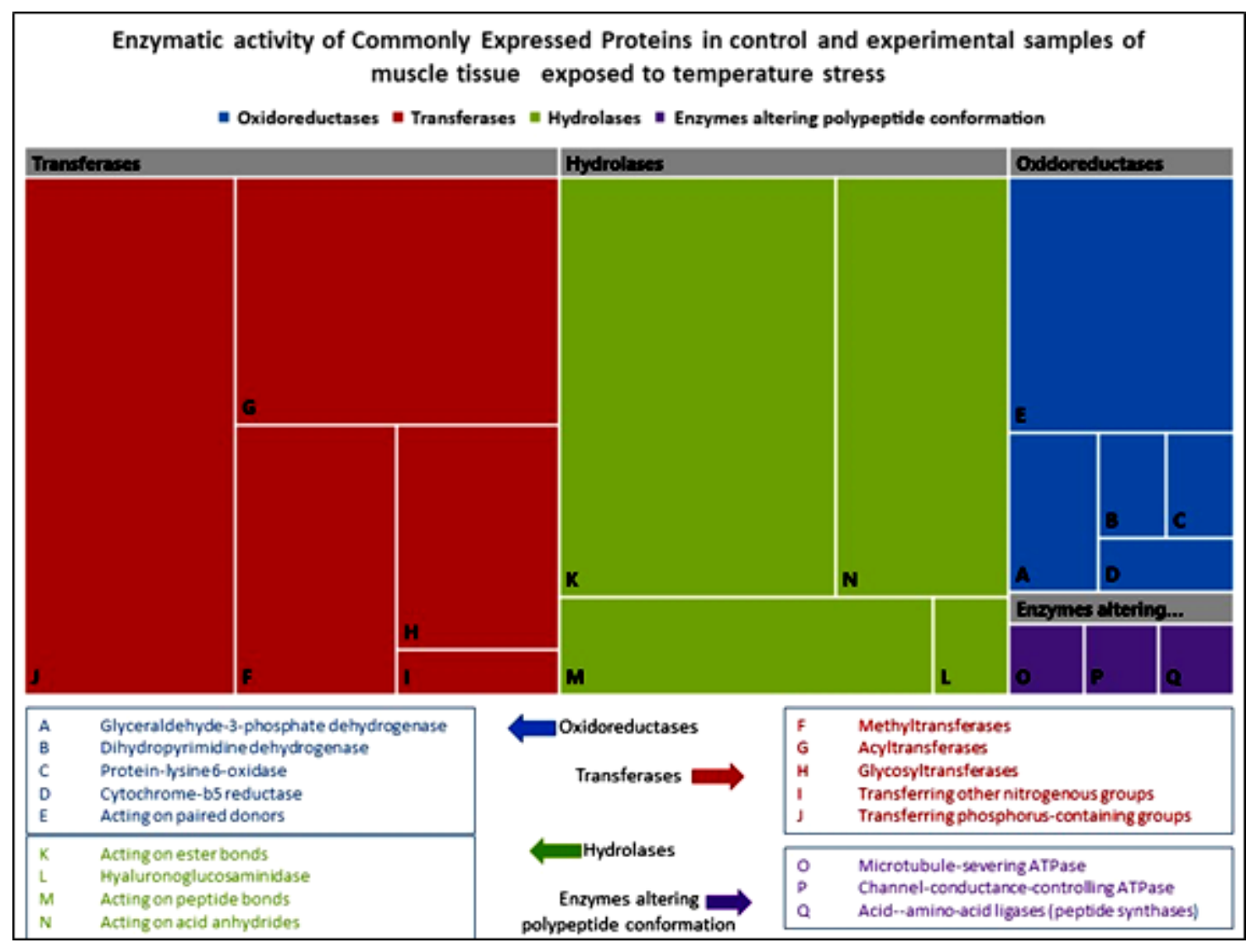

Figure 4

Enzymatic activity of 275 Commonly Expressed Proteins in control and experimental samples of muscle tissue exposed to temperature stress

Pathway analysis of Commonly Expressed Proteins in control and experimental samples of muscle tissue exposed to temperature stress

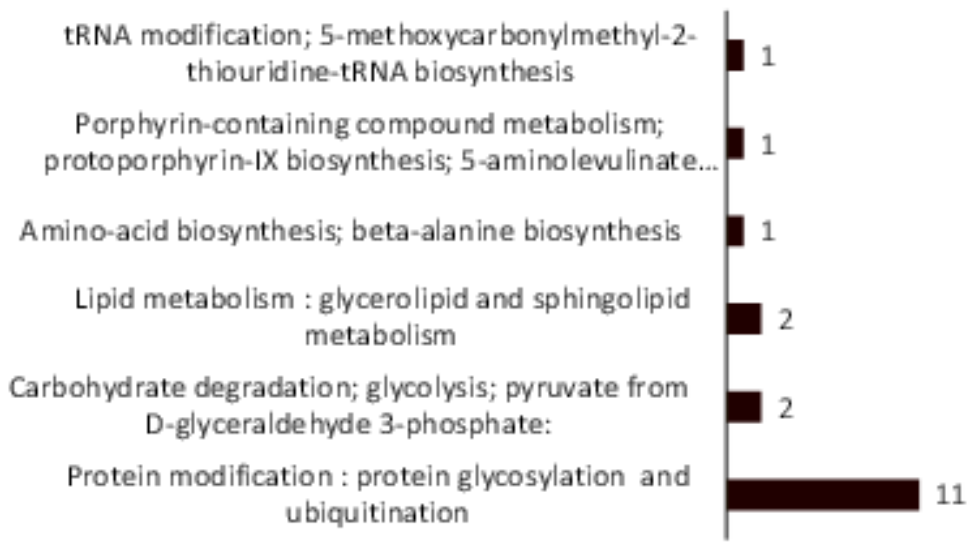

\section{Figure 5}

Pathway analysis of 275 Commonly Expressed Proteins in control and experimental samples of muscle tissue exposed to temperature stress 


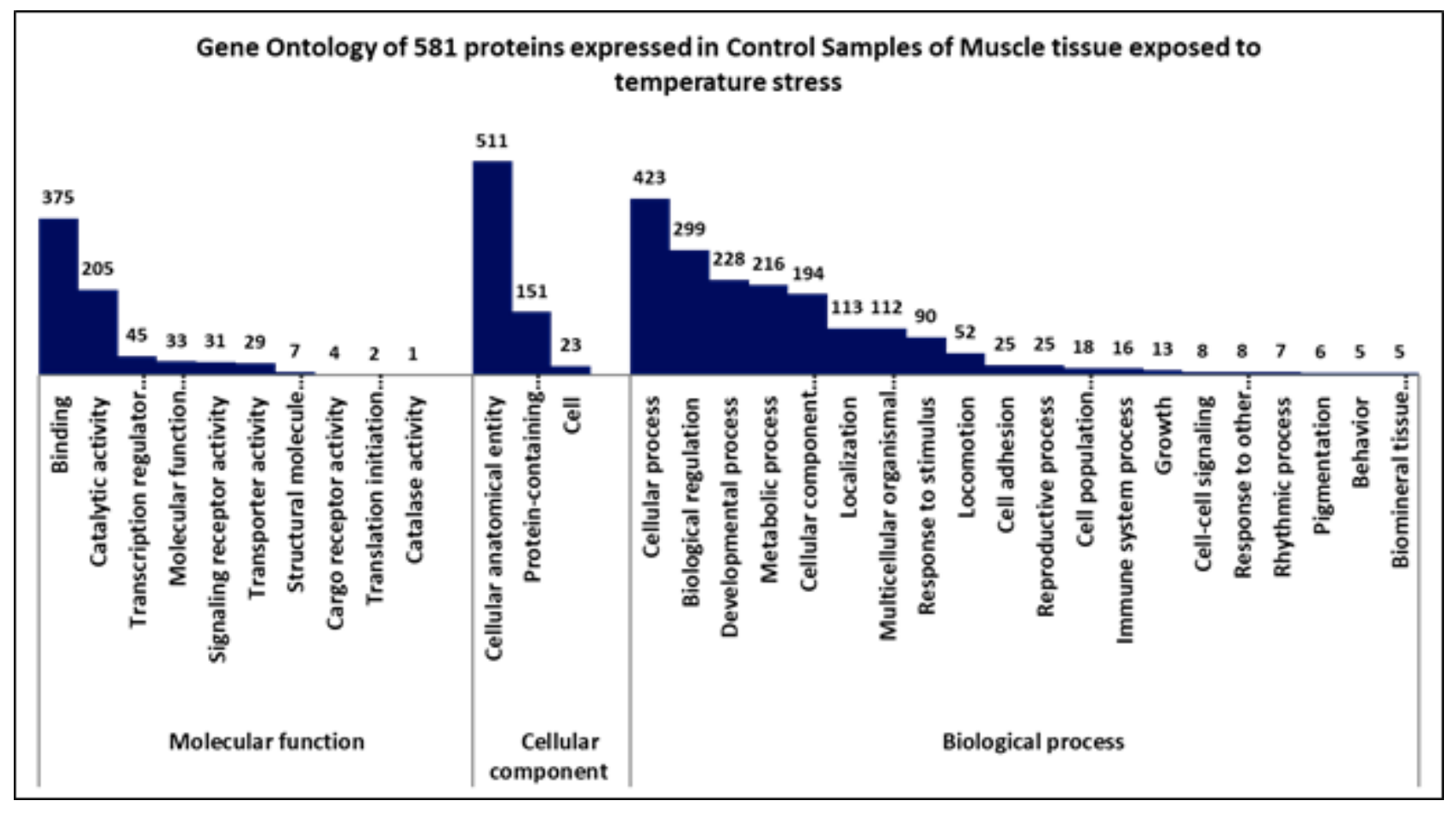

Figure 6

Gene Ontology of 581 proteins expressed in Control Samples of Muscle tissue

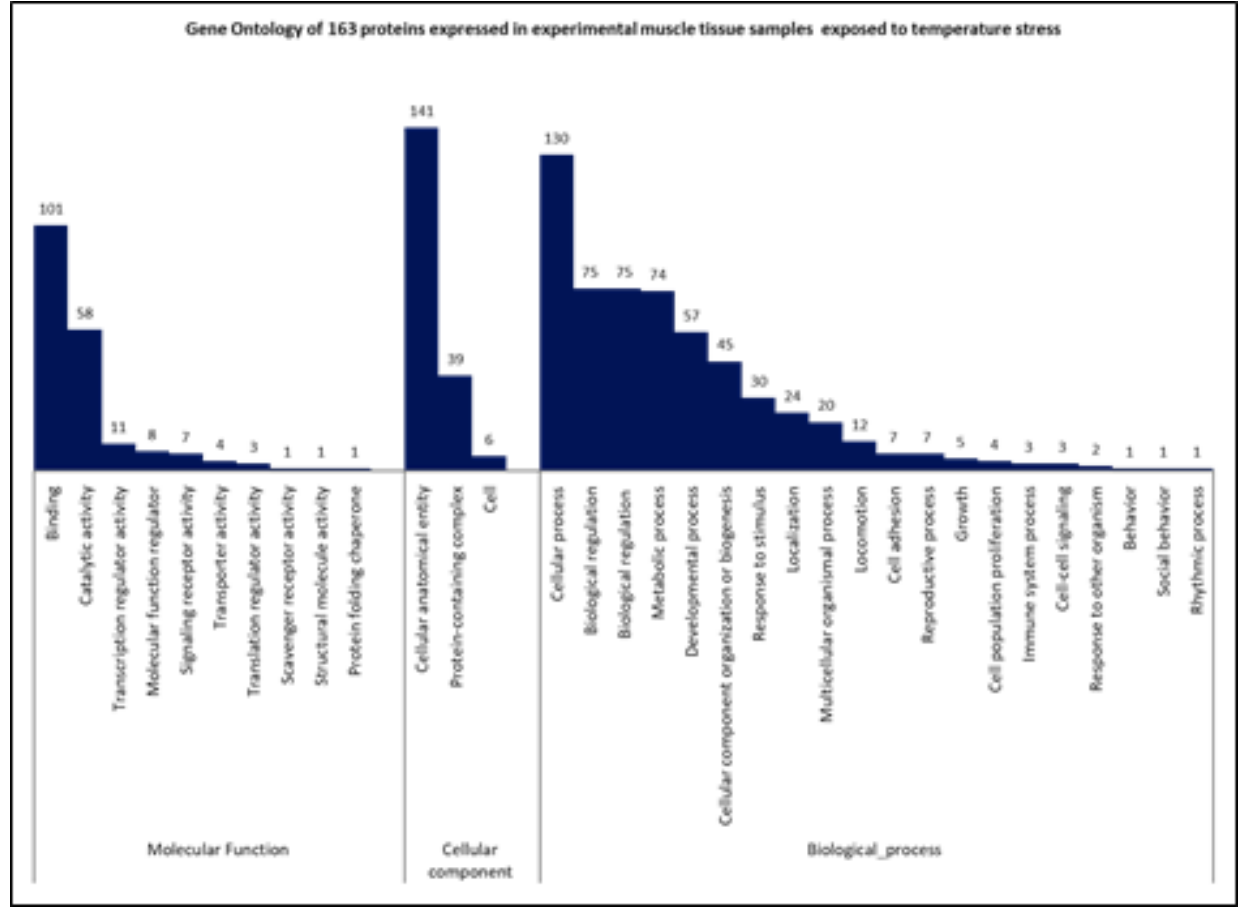

Figure 7

Gene ontology of 163 proteins expressed in experimental samples of muscle tissue exposed to temperature stress 


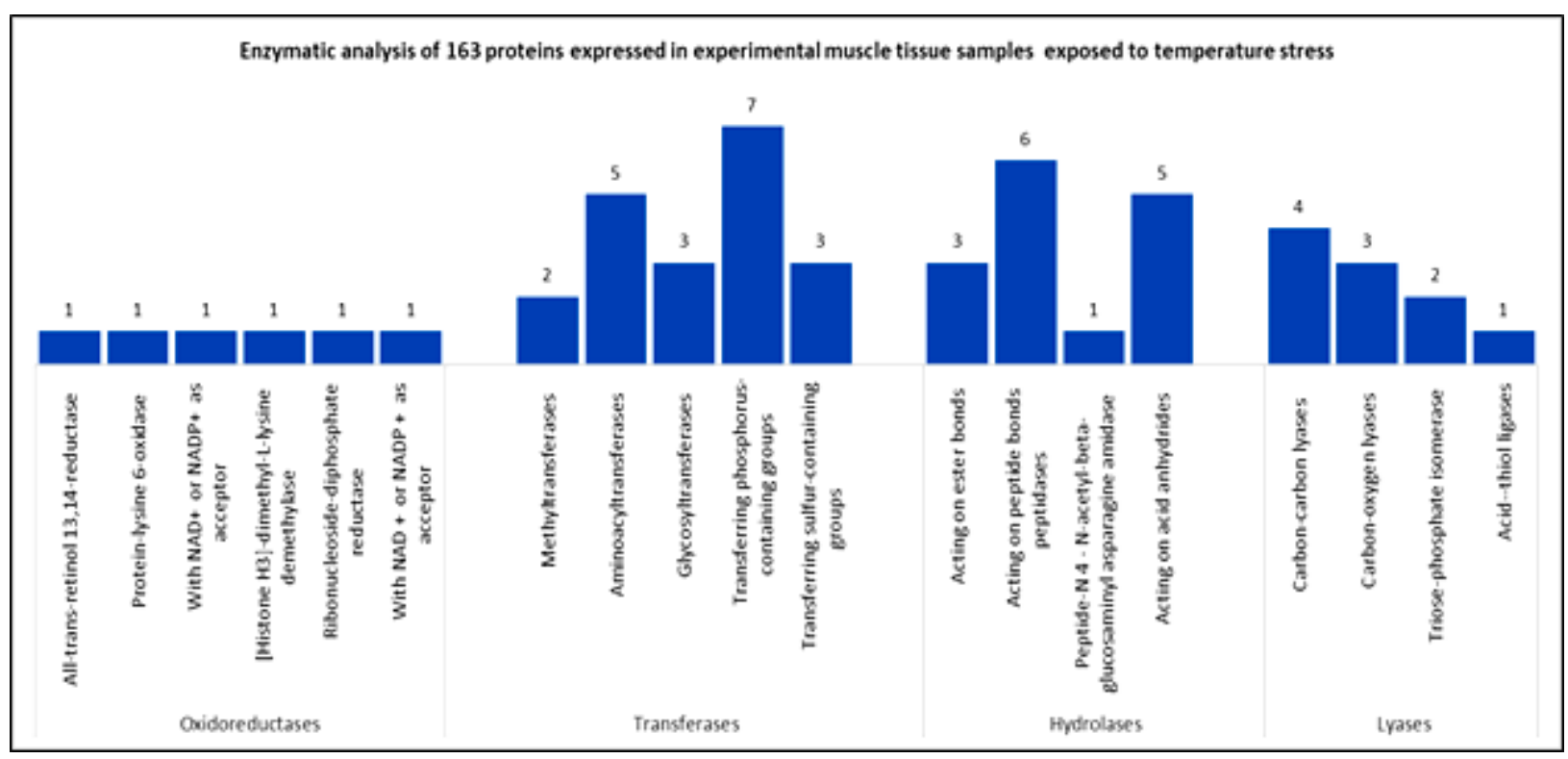

Figure 8

Enzymatic analysis of 163 proteins expressed in experimental muscle tissue samples exposed to temperature stress

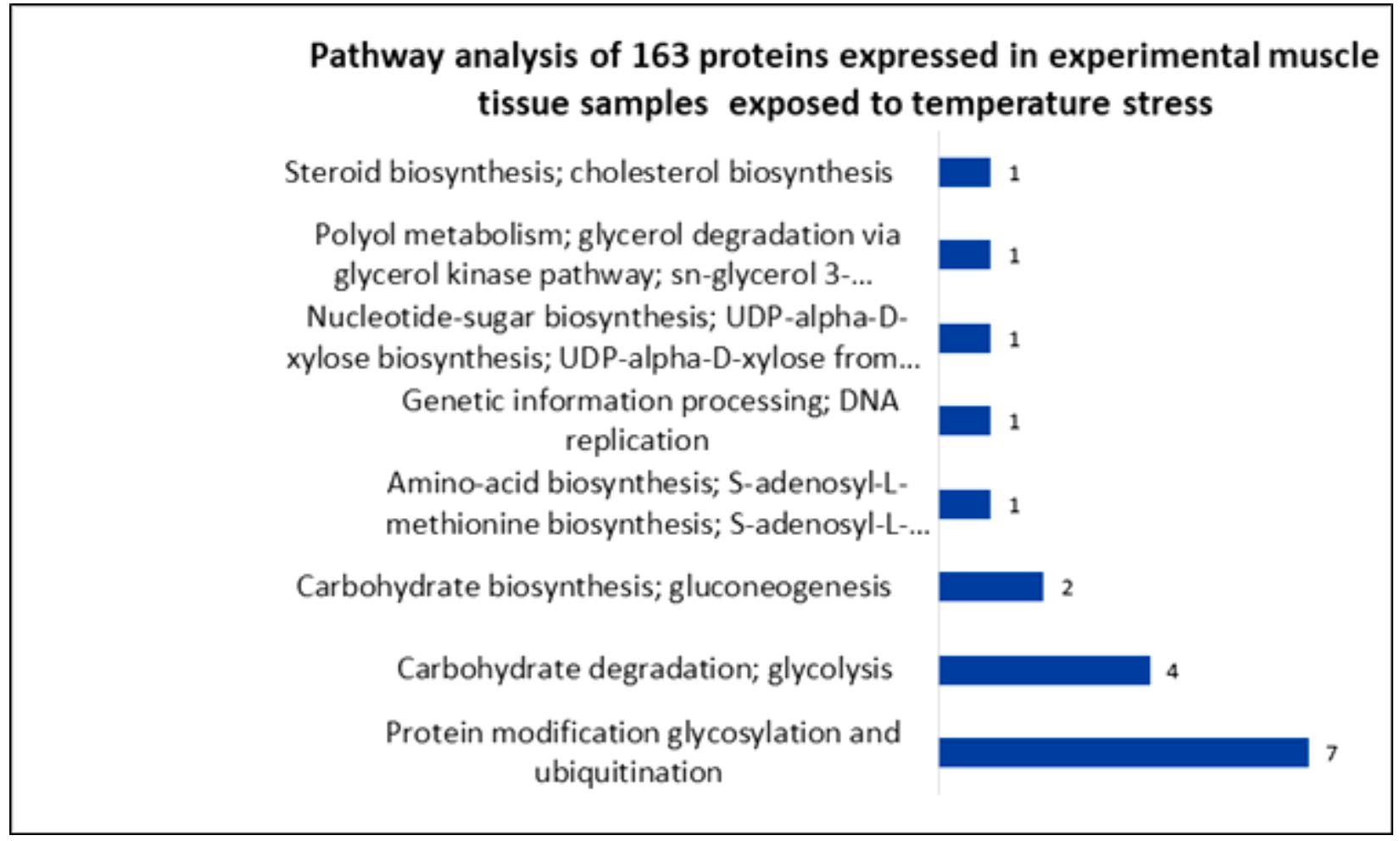

Figure 9

Pathway analysis of 163 proteins expressed in experimental muscle tissue samples exposed to temperature stress

\section{Supplementary Files}


This is a list of supplementary files associated with this preprint. Click to download.

- floatimage1.jpeg 